\title{
Tradiciones alfareras, identidad social y el concepto de etnias tardías en Conchucos, Ancash, Perú
}

Traditions céramiques, identité sociale et le concept d'ethnie au Conchucos

Ceramic traditions, social identity and the concept of ethnic groups in Conchucos

Isabelle Druc

\section{(2) OpenEdition \\ Journals}

Edición electrónica

URL: http://journals.openedition.org/bifea/2853

DOI: 10.4000/bifea.2853

ISSN: 2076-5827

Editor

Institut Français d'Études Andines

Edición impresa

Fecha de publicación: 1 abril 2009

Paginación: 87-106

ISSN: 0303-7495

Referencia electrónica

Isabelle Druc, "Tradiciones alfareras, identidad social y el concepto de etnias tardías en Conchucos, Ancash, Perú », Bulletin de l'Institut français d'études andines [En línea], 38 (1) | 2009, Publicado el 01 octubre 2009, consultado el 10 diciembre 2020. URL : http://journals.openedition.org/bifea/2853 ; DOI : https://doi.org/10.4000/bifea.2853

\section{(c)}

Les contenus du Bulletin de l'Institut français d'études andines sont mis à disposition selon les termes de la licence Creative Commons Attribution - Pas d'Utilisation Commerciale - Pas de Modification 4.0 International. 


\title{
Tradiciones alfareras, identidad social y el concepto de etnias tardías en Conchucos, Ancash, Perú*
}

\author{
Isabelle Druc**
}

\section{Resumen}

Este artículo trata de identificar los grupos humanos vinculados históricamente con las tradiciones cerámicas observadas en Conchucos. La discusión enfoca al grupo Huari, la noción de grupo étnico, las parcialidades y lo que estas divisiones sociopolíticas representan mediante el análisis de las prácticas cerámicas. El examen de las tecnologías cerámicas, de la división del trabajo por categorías sexuales y del reparto geográfico de estas tradiciones revelan una nueva imagen de la composición de los grupos sociopolíticos en el Intermedio Tardío en Conchucos sur y central.

Palabras clave: tradición cerámica, grupos étnicos, etnohistoria, Conchucos, Ancash, Perú

\section{Traditions céramiques, identité sociale et le concept d'ethnie au Conchucos}

\section{Résumé}

Cet article tente d'identifier les groupes humains qui, historiquement, sont reliés aux traditions céramiques observées dans le Conchucos. La discussion porte sur le groupe Huari, la notion de groupe ethnique, de parcialités et sur ce que ces divisions socio-politiques représentent en fonction de l'analyse des pratiques céramiques. L'examen des technologies céramiques, de la division sexuelle du travail et de la distribution géographique de ces traditions donne une image nouvelle de la composition des groupes socio-politiques à I’Intermédiaire Récent au Conchucos sud et central.

Mots clés : Tradition céramique, groupes ethniques, ethnohistoire, Conchucos, Ancash, Pérou

* Una primera versión de este artículo fue presentada en la $35^{\text {th }}$ Annual Midwest Conference on Andean and Amazonian Archaeology and Ethnohistory, in Carbondale, Illinois (febrero 2007), bajo el título: Ceramic Traditions and Ethnohistorical Boundaries. Do they Match? A case from Conchucos, Ancash, Peru.

** Department of Anthropology, University of Wisconsin-Madison. E-mail: idruc@mhtc.net 


\title{
Ceramic traditions, social identity and the concept of ethnic groups in Conchucos
}

\begin{abstract}
This article explores the issues of social identity, Late Intermediate Period ethnic groups and sociopolitical divisions in relation to the ceramic traditions observed in southern and central Conchucos. Based on differences in ceramic technology, gendered production and distribution of the ceramic traditions, two sociocultural groups are identified and associated with different factions of the Huari group.
\end{abstract}

Keywords: Ceramic Tradition, Ethnic Groups, Ethnohistory, Conchucos, Ancash, Peru

\section{INTRODUCCIÓN}

Sin quererlo, el estudio de las tradiciones contemporáneas de Conchucos, iniciado en el año 1994 como una investigación etnoarqueológica me ha llevado a un viaje etnohistórico y teórico acerca de la relación entre producción cerámica y grupos étnicos.

La primera conclusión del estudio inicial indica que en Conchucos existen dos tradiciones cerámicas distintas (Druc, 2000; 2001; 2005). Una tradición, femenina, utilizando el enrollado como sola técnica de elaboración, está vinculada a la parte sur de Conchucos (actual provincia de Huari) (fig. 1). La otra tradición, masculina, caracterizada por el uso del paleteado como técnica secundaria de elaboración, se observa en la zona central en la cuenca sur del río Yanamayo (provincias de Asunción, Fermín Fitzcarrald y Antonio Raimondi) (fig. 2).

La segunda conclusión del estudio indica que estas tradiciones, en particular su tecnología, fueron poco alteradas por la presencia inca y colonial (Druc, 2005). No consta, para
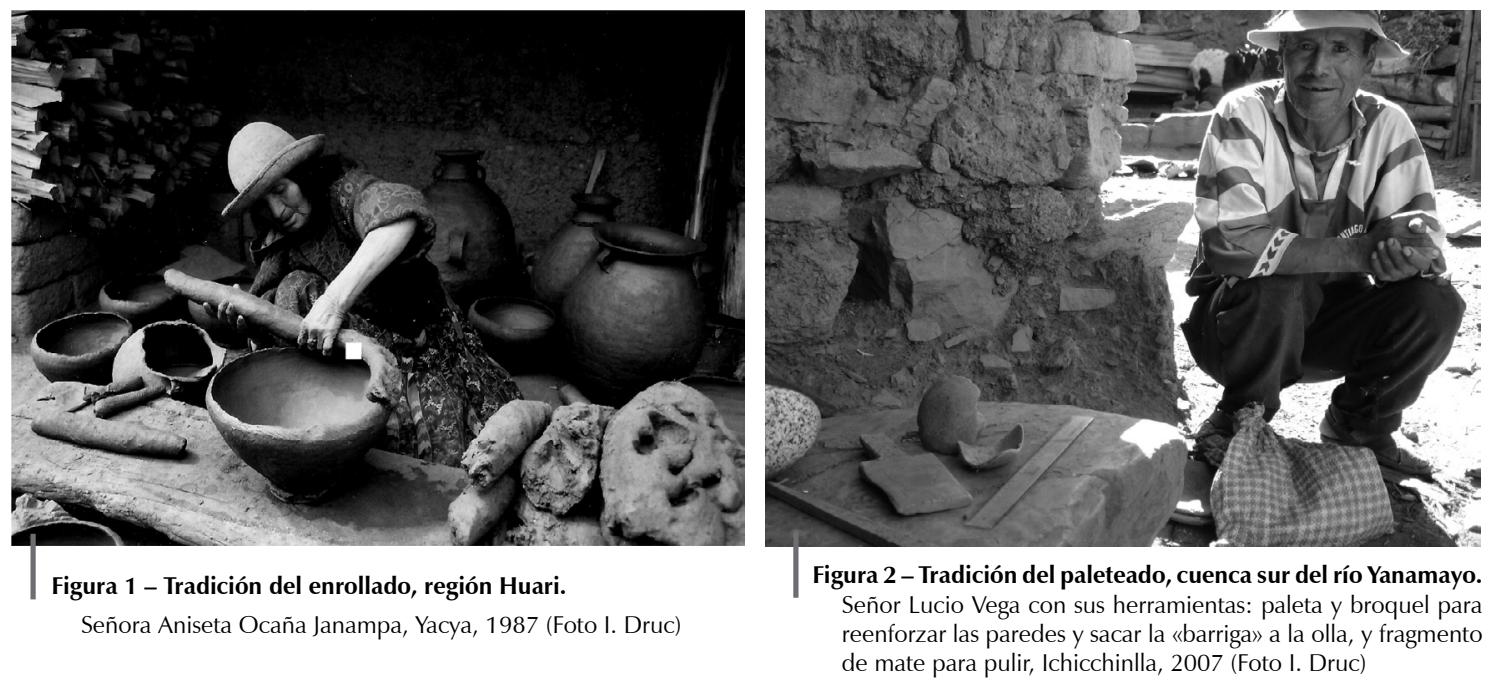
Conchucos sur y central, con la implementación de talleres de producción cerámica tanto incas como españoles, o con la producción de cerámica inca o colonial según las normas técnicas y formales características para estas producciones (D'Altroy et al., 1994; Hayashida, 1998; Acevedo, 2004). Por lo tanto, la cerámica continuó produciéndose para la población local como antes, como se observó también para otras regiones del Perú (Hagstrum, 1987; Lavallée, 1967; Tschopik, 1950).

Tampoco se menciona en los documentos etnohistóricos el traslado por los incas de alfareros de otras provincias a Conchucos sur o central. No hablo aquí del traslado de otros tipos de mitimaes que sí ocurrió en Conchucos. En otras regiones de Ancash, sí, alfareros fueron trasladados, como en la provincia de Recuay, al sur del Callejón de Huaylas (Duviols, 1986; Hernández Príncipe, 1923). Pero allí también se puede argumentar que la tecnología cerámica no fue alterada. Los alfareros en cuestión provenían de la misma provincia, según el nombre del lugar de origen dado en el trabajo de Pierre Duviols (1986: 492-493, 504), una costumbre común en este tiempo (Hayashida, 1998). Lo que sugiere que estos alfareros mitimaes eran portadores de una tradición cerámica similar a la tradición del lugar de relocación. El análisis de la relación de Hernández Príncipe por Tom Zuidema (1989: 132), sin embargo, menciona dos grupos de alfareros de dos regiones diferentes para el ayllu de Olleros. En este caso, habría que investigar de qué regiones se habla.

Acerca de la zona de estudio, había pensado inicialmente que siendo preincas las dos tradiciones observadas correspondían a expresiones de las naciones o grupos Huari y Piscobamba, en la zona sur y central de Conchucos, respectivamente (Druc, 2005). Sin embargo, esta última conclusión tiene que ser revisada a la luz de un análisis más detallado tomando en cuenta los documentos etnohistóricos, la distribución de los pueblos alfareros en Conchucos sur y central, y la topografía.

\section{ORGANIZACIÓN CONCEPTUAL DEL TRABAJO}

El presente trabajo es un estudio sociohistórico, basado en el análisis de las prácticas alfareras. Estas prácticas trianguladas a los datos geográficos y etnohistóricos para Conchucos apuntan a dos grupos socioculturales distintos en la zona de estudio. El objetivo último es identificar los grupos humanos históricamente en relación con las tradiciones cerámicas observadas. A este fín, es necesario examinar la composición y la organización sociopolítica de estos grupos.

Empezaré con una presentación del cuadro conceptual utilizado en relación con las unidades sociales básicas y los grupos étnicos en Conchucos, para luego proponer marcadores de identidad étnica que alumbran las diferencias cerámicas entre Conchucos sur y central, discutiendo el cuadro conceptual relacionado a los conceptos de tecnología e identidad étnica. Seguiré enfocando la discusión hacia el grupo Huari, la noción de parcialidad y lo que estas divisiones sociopolíticas representan mediante el análisis de las tradiciones cerámicas. Terminaré con una perspectiva diacrónica poniendo en paralelo la evolución de estas dos tradiciones con los grupos humanos a los cuales se relacionan.

El cuadro sociohistórico utilizado aquí para investigar la realidad sociopolítica en Conchucos al torno de la conquista española toma en cuenta las definiciones y discusiones de María Rostworowski, Gerald Taylor y Carolina Orsini acerca de grupos étnicos, ayllus, Ilactas y parcialidades (o facciones). Se entiende la palabra étnico en un sentido mítico histórico y no biológico. Una vez definidas estas nociones, podremos pasar a un nivel superior de interpretación de los datos, poniendo en relación identidad y estilo, según un cuadro conceptual derivado de André Leroi-Gourhan y James Sackett. 


\section{UNIDADES BÁSICAS Y GRUPOS ÉTNICOS EN CONCHUCOS}

El estudio de Miguel León Gómez (2003) sobre el espacio geográfico y la organización social de los grupos étnicos en Conchucos en los siglos XVI y XVII nos permite discutir el problema de identidad social en esta región. Basándose en documentos coloniales, este autor nota la presencia de sur a norte de los siguientes grupos: pincos, huari, piscobamba, siguas y conchucos (fig. 3). De la composición de estos grupos no se sabe mucho, sino que los grupos pincos y huari fueron divididos en dos parcialidades, constituidas de varias pachacas cada una (León Gómez, 2003). Según León Gómez (2003: 458), la pachaca forma la unidad étnica básica en Conchucos, también llamada ayllu en documentos administrativos de los siglos XVI y XVII, o simplemente conocida bajo el nombre del pueblo. Aunque una pachaca solía designar a grupos de 100 familias en la organización sociopolítica inca, el número de familias, es decir, la composición de pachacas en el norte del Perú (incluido Ancash) es muy relativa (Espinoza Soriano, 1978; Rostworowski, 1981) y puede aludir a grupos menos numerosos.

El término ayllu en este contexto debe entenderse en el sentido de líneas de descendencia ceremonial (Zuidema, 1989: 132), pero no como grupo de parientes.

Esta equivalencia entre pachaca y ayllu, la menciona también Maria Rostworowski (1996: 140). Además, esta autora relata que

«existía la noción indígena muy clara de micro-etnias regionales» (Rostworowski, 1996: 122).

No define los atributos de la microetnia pero se entiende que lengua, trajes y tocados son elementos diferenciales.

\section{MACROETNIA}

A un estado de organización sociopolítica más alto suele encontrarse la macroetnia, según María Rostworowski (1990). Esta autora propone que un curacazgo, para acceder al rango de macroetnia, suele demostrar los siguientes elementos básicos (Rostworowski, 1990: 16):

1) unidad de origen y creencias;

2) unidad de lengua o dialecto;

3) unidad de atuendo;

4) unidad sociopolítica.

Así, el concepto de macroetnia como organización de mayor rango, no es nada más que una ampliación de la microetnia, que se expandió o se unió a otros grupos de misma filiación mítica (original o adoptada). El concepto o la definición para «macroetnia〉 propuesta por Rostworowski se refiere a una organización sociopolítica englobante, formada de grupos básicos, las pachacas, que muestran elementos de composición similares, es decir, los puntos 1 a 3 arriba mencionados.

Sin embargo, el concepto de macroetnia no funciona para una macro organización pluriétnica, formada de grupos de individuos con diferentes creencias, origenes y/o lenguas. Ya en 1991, Gary Urton cuestionaba la unidad composicional de los señoríos étnicos o reinos (Urton, 1991). Veremos aquí que este término no puede aplicarse al caso del grupo Huari.

León Gómez duda también de la presencia de macroetnias en Conchucos (2003). Desde luego él hace uso del término de grupo étnico. Sin embargo, uno puede preguntarse si 


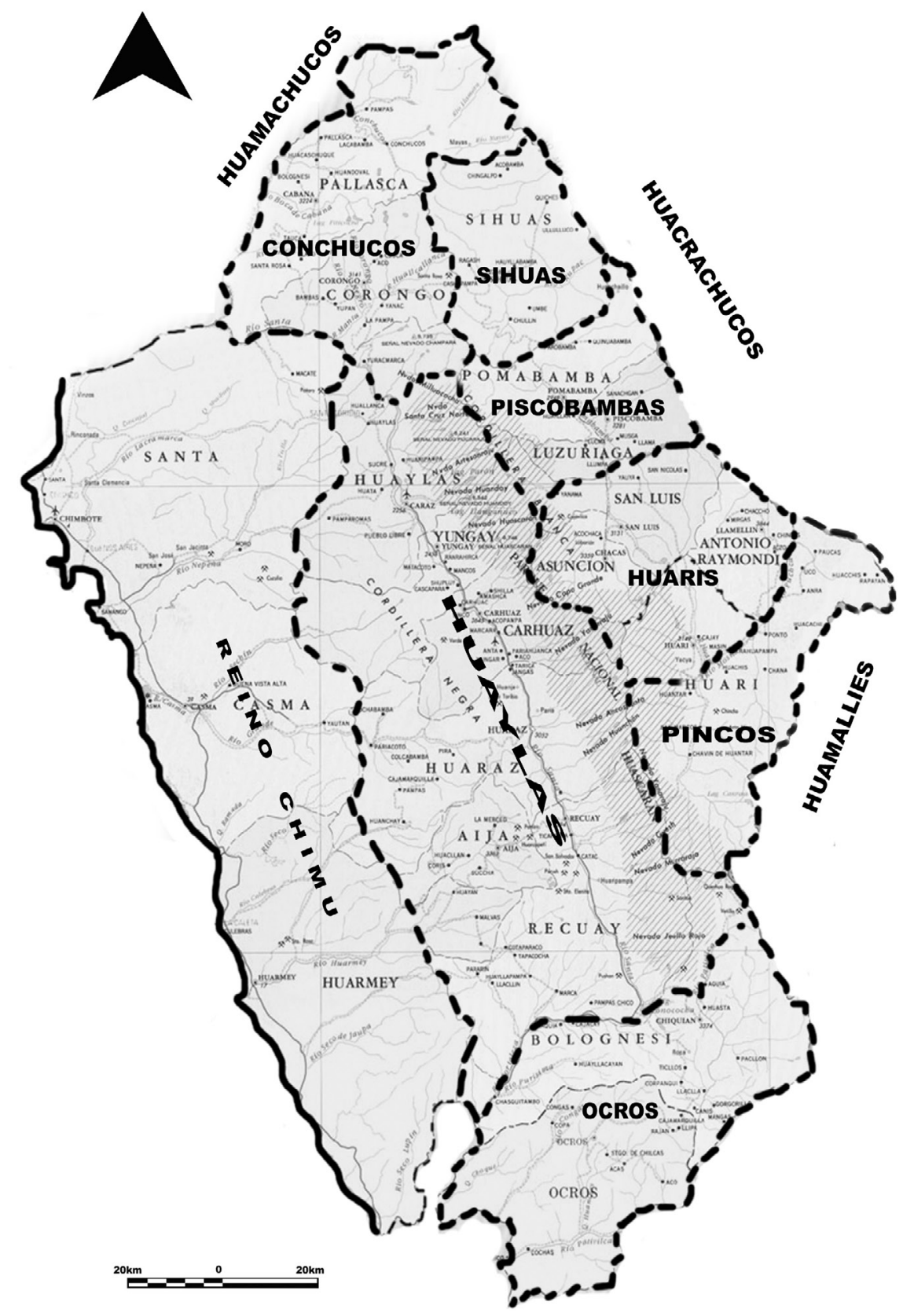

Figura 3 - Mapa de las provincias de Ancash y grupos étnicos al tiempo de la conquista inca. Redibujado por Bebel Ibarra a partir del mapa físico-político del departamento de Ancash. Instituto Geográfico Nacional del Perú, 1992. Adaptado de Druc, 2005: figura 7 
este término se aplica bien al grupo huari. «Grupo étnico» refleja una composición humana homogénea, según el concepto definido por Rostworowski para los Andes (1990), es decir un grupo humano descendiente del mismo ancestro, con el mismo origen, creencia, lengua y atuendo, poblando un dicho territorio.

Este concepto difiere del concepto de ayllu como grupo político expresado por Tom Zuidema (1989) en cuanto a la homogeneidad de origen y creencias. Se relaciona más bien con la organización política de los grupos humanos en ayllus locales en organizaciones jerárquicas siempre más grandes, también llamadas ayllus (Zuidema, 1989: 96).

Falta ahora examinar más en detalle el grupo huari para enmarcarlo en el contexto histórico.

\section{EL GRUPO HUARI Y LA NOCIÓN DE PARCIALIDAD}

En los documentos coloniales, el grupo huari se presenta dividido en dos parcialidades llamadas Ichoc Huari y Allauca Huari (León Gómez, 2003). La noción de parcialidad, explorada por Rostworowski (1981), está definida como una división sociopolítica de los ayllus agrupados en bandos:

«El ayllu era una unidad de parentesco unida por un origen común y mítico. En cambio el suyu o parcialidad indicaba las divisiones sociopolíticas de los varios ayllus agrupados en bandos» (Rostworowski, 1981: 43).

Rostworowski precisa en otra parte que los bandos son equivalentes a parcialidades (1996: 115).

León Gómez presenta las dos parcialidades de huari como un solo grupo étnico (2003: 459), sugiriendo que las dos parcialidades tenían un origen mítico común y —supuestamentecostumbres similares. Sin embargo, en una ocasión, León Gomez equivale las parcialidades de Ichopincos, Ichohuari y Allaucahuari a grupos étnicos distintos:

«consignamos el nombre de algunas de las pachacas... con su correspondiente grupo étnico» (2003: 460).

¿Qué suele ser? Puede ser que la confusión se origine en los documentos coloniales. Thierry Saignes (1991) advierte que diferentes grupos étnicos han podido ser clasificados por los administradores españoles bajo el mismo nombre. Hasta que se clarifique este punto, se puede utilizar el término más neutral de nación para Huari. Lleva nociones de territorialidad y lugar de nacimiento, y la aceptación moderna del término no implica necesariamente un mismo origen étnico (Salazar, 1998).

Es importante precisar que había también «pequeños curacazgos que no representaban distintas etnias sino el sistema organizativo andino» (Rostworowski, 1990: 19). Asimismo, Rostworowski llama la atención sobre el peligro de categorizar como «etnia» cada división o señorío (1990). De allí otra advertencia, la de Carolina Orsini (2006) quien observa que los grupos culturales o políticos mencionados en los documentos coloniales no son necesariamente expresiones válidos de grupos étnicos. Orsini analiza la oposición entre huari y llacuaz con una nueva perspectiva y propone ver la oposición entre las facciones del grupo huari como un reflejo de la posición social más que como divisiones étnicas (Orsini, 2006: 159). Argumenta que una diversidad económica, como entre pastores y agricultores, no siempre refiere a grupos étnicos distintos. La economía básicamente agropecuaria de toda la región, con acceso a las mismas ecozonas, no justifica una oposición a base de complementariedad vertical. Orsini propone más bien una oposición a base del prestigio social concedido a cada facción en relación con la economía favorecida como la cría de ganado para los llacuaz (Orsini, 2006: 159). 
La discusión basada en la oposición entre llacta y llacuaces expuesta por Hernández Príncipe en 1622 (Zuidema, 1973), y analizada por Pierre Duviols (1973)1, nos lleva al concepto de llacta como concepto alternativo al grupo étnico2.

Una definición de llacta se encuentra en la obra de Gerald Taylor (1980: 13) sobre el manuscrito de Huarochirí, en la sierra de Lima. Taylor relaciona llacta con comunidad, población, espacio y huacas. Es decir, la relaciona con el grupo humano, su filiación mítica, sus creencias y su hábitat. La unidad de la comunidad está establecida por el reconocimiento de un protector o huaca supremo (Taylor, 1980: 12). En su sentido general, Ilacta refiere a todos los aspectos que asocian un grupo humano a su lugar de origen mítico3, el territorio, la deidad protectora y su santuario. Una llacta (como ancestro protector y comunidad) puede también dominar un territorio extenso y tener una amplitud supra-comunitaria (Taylor, 1980: 14). Esta definición se acerca a la de Rostworowski, sin el peso negativo asociado al vocablo etnia en relación con una noción biológica o racial (Abercrombie, 1991). Para Zuidema, varias llactas pueden conformar un ayllu, aludiendo a un grupo o a una organización de rango inferior a este (1989: 132).

Entonces, ¿A qué tipo de grupo se refieren las parcialidades de Huari? ¿A curacazgos que se unieron para formar la nación huari? ¿A llactas? ¿A ayllus o a comunidades socioeconómicas?

\section{OPERACIONALIDAD DEL CONCEPTO DE IDENTIDAD}

Los conceptos previamente presentados están íntimamente ligados a nociones míticoterritoriales, de parentesco o económicas. Para distanciarnos del debate acerca del grupo étnico, debemos buscar una definición más operacional para nuestro asunto. El cuadro conceptual que utilizo pone en relación etnicidad y estilo. Deriva de la definición de etnia dada por Leroi-Gourhan (1988) y del concepto de isochrestic variation definido por James Sackett (1990: 33). La definición de estilo como una forma no verbal de comunicación, haciendo algo de un cierto modo que comunica informaciones acerca de la identidad del actor (Wiessner, 1990: 107), refuerza la idea discutida aquí. Esta definición está propuesta por Wiessner (1990: 107) como concepto de trabajo para las investigaciones arqueológicas.

La dimensión cultural e histórica de la formación de la identidad social está expuesta por Margaret Wetherell (1982) quien argumenta que los diversos modos de identificarse (como grupo distinto del otro) están culturalmente e históricamente determinados (in: Wiessner, 1990: 107). Por último, Heather Lechtman presenta en 1977 el concepto de technological style (estilo tecnológico o técnico). Este concepto está vinculado a la caracterización de objetos tomando en cuenta la elección de materias primas, elementos y técnicas dentro de ciertos contextos sociopolíticos.

En cuanto al término de etnia, fue utilizado mucho en los años 1970 como sinónimo de grupo cultural (Cohen, 1978). Sin embargo, la definición dada por André Leroi-Gourhan lleva elementos operativos que permiten enfocar la discusión hacia una definición arqueológica, que incluye la cultura material, y por lo tanto la cerámica. Así, Leroi-Gourhan (1988: 386) declara:

Es interesante notar que Gerald Taylor opone llactas (moradores oriundos) a advenedizos (nuevos habitantes por inmigración, invasión, casamiento o adopción) (Taylor, 1980: 14).

2 Agradezco a Frank Salomon por indicarme esta pista de reflexión.

3 La versión francesa precisa: Il s'agit donc de la déité qui protège et anime la communauté, du sanctuaire de cette déité, des terres qu'elle anime et protège et de la communauté qui y habite (Taylor, 1980: 13). 
«... los comportamientos humanos se constituyen a lo largo de un aprendizaje que se hace en el seno de un grupo de hombres que realiza una manera de ser humano y que es designado con el nombre de etnia... los índices más seguros de identidad étnica son la afirmación por los individuos de su pertenencia a esta etnia, su comunidad lingüística y esta "manera propia de asumir y de marcar las formas, los valores y los ritmos" que constituyen el estilo étnico. En la ausencia de los dos primeros marcadores, la cultura material de las poblaciones estudiadas por los arqueólogos sirve de base para la identificación étnica» (traducido del original en francés) 4 .

\section{ESTILO Y TECNOLOGÍA COMO MARCADORES DE IDENTIDAD ÉTNICA}

En la definición de Leroi-Gourhan, el enfoque hacia el estilo étnico es importante. En muchos casos, el estilo cerámica (diseños, acabado, formas) constituye un carácter mayor de identificación de grupos culturales en las muestras arqueológicas, además de uso cronológico. Se estima que el estilo resulta de una elección y marca la identidad étnica mejor que otros elementos de la cultura material basados en apremios técnicos o funcionales. Por ejemplo, la misma jarra, la misma forma, puede ser decorada de maneras distintas. La elección de los elementos decorativos normalmente responde a normas vigentes en el grupo, en la cultura propia del alfarero o pintor responsable de la decoración de la pieza. Esta reflexión se aplica también a la tecnología adoptada.

Según James Sackett, los artesanos tienen conciencia de algunas de las opciones posibles para llevar a cabo sus tareas, pero muchas veces eligen solamente una manera, siendo esta elección dictada por las tradiciones tecnológicas dentro de las cuales están esculturados como miembros de un grupo social que delinea su etnicidad (1990: 33). Olivier Gosselain elabora sobre el mismo concepto, llamando tradiciones técnicas el conjunto de convenciones y costumbres transmitidas por aprendizaje, enfatizando en el estilo técnico, las nociones de elección, herencia y sentido. Argumenta que una valorización de los estilos técnicos permite una mejor aproximación de las estructuras sociales, de la etnicidad y de las fronteras culturales. Recomienda tomar en cuenta los varios parámetros de identidad social, como estilos técnicos, formales y ornamentales, para una definición más realista de la identidad y de las fronteras sociales (Gosselain, 2002: 10-11).

Desde luego, considero la tecnología como un marcador de identidad étnica. Responde al isochrestic model (modelo isocréstico) de Sackett que explica el enlace entre estilo y etnicidad en los productos formales, como la elección de una alternativa dentro de todas las alternativas posibles para elaborar o usar un artículo (1990: 33). Un alfarero puede emplear diferentes técnicas de producción y la tecnología puede resultar de necesidades funcionales o formales. Así, la pasta puede variar según la pieza a producir; jarras muy grandes requieren otra técnica de manufactura que la del torno, y el acabado difiere si el producto sirve para contener líquidos o sólidos (Velde \& Druc, 1999). A pesar de esto, el uso de una tecnología particular (como el paleteado en vez del golpeado) corresponde a

4 Leroi-Gourhan, 1988: 386: «... les comportements humains se constituent au cours d'un apprentissage, qui se fait au sein d'un groupe d'hommes qui réalise une des façons d'être humain et que l'on désigne sous le nom d'ethnie... les indices les plus sûrs de l'identité ethnique sont l'affirmation par les individus de leur appartenance à cette ethnie, leur communauté linguistique et cette "manière propre d'assumer et de marquer les formes, les valeurs et les rythmes" qui constitue le style ethnique. En l'absence des deux premiers marqueurs, la culture matérielle des populations étudiées par les archéologues sert de base pour l'identification ethnique». 
una elección y a un aprendizaje que se hace en el seno de un grupo sociocultural. De igual modo veo lo oposición entre oficio masculino y femenino como un marcador de identidad étnica o sociocultural.

La distribución de las tradiciones cerámicas refuerza este concepto de identidad de un grupo humano expresada en la tecnología y en el género del oficio. En Conchucos, la distribución de las dos tradiciones estudiadas se delimita geográficamente, y las características observadas no se mezclan ni se superponen.

A modo de ilustración, presento el cuadro sintético de las diferencias observadas entre las tradiciones alfareras en Conchucos sur y central.

Cuadro 1 - Resumen de las características de las tradiciones cerámicas en Conchucos sur y central

\begin{tabular}{|c|c|c|}
\hline & $\begin{array}{l}\text { Conchucos sur } \\
\text { (región de Huari) }\end{array}$ & $\begin{array}{l}\text { Conchucos central } \\
\text { (Cuenca del Yanamayo) }\end{array}$ \\
\hline Ocupación & $\begin{array}{l}\text { femenina con ayuda de los } \\
\text { hombres para obtener las } \\
\text { materias primas y hacer la quema }\end{array}$ & Masculina \\
\hline Pasta & $\begin{array}{l}\text { Raaku (arcilla) }+ \\
\text { Shashal (temperante de pizarra } \\
\text { molida) }\end{array}$ & $\begin{array}{l}\text { Raaku (arcilla) }+ \\
\text { Shashal (temperante de } \\
\text { pizarra molida) }\end{array}$ \\
\hline Manufactura & Enrollado & Enrollado + paleteado \\
\hline $\begin{array}{l}\text { Técnica del } \\
\text { paleteado }\end{array}$ & Ausente & Presente \\
\hline Plato alfarero & $\begin{array}{l}10-12 \mathrm{~cm} \text {. de diámetro, con o sin } \\
\text { base anular }\end{array}$ & $\begin{array}{l}14-16 \mathrm{~cm} \text {. de diámetro sin } \\
\text { base anular }\end{array}$ \\
\hline Quema & $\begin{array}{l}\text { En hoyo poco hondo. Quema } \\
\text { hecha por un especialista, con } \\
\text { achupalla, paja y/o bosta de } \\
\text { animales. }\end{array}$ & $\begin{array}{l}\text { En el suelo, con madera } \\
\text { y paja. A veces con } \\
\text { achupalla. } \\
\text { Hecha por el alfarero } \\
\text { mismo. }\end{array}$ \\
\hline $\begin{array}{l}\text { Comunidades } \\
\text { alfareras }\end{array}$ & Yacya, Mallas, Acopalca & $\begin{array}{l}\text { Chinlla, Ichicchinlla y } \\
\text { alrededores }\end{array}$ \\
\hline
\end{tabular}

\section{DISTRIBUCIÓN DE LAS TRADICIONES ALFARERAS EN CONCHUCOS SUR Y CENTRAL}

Hemos visto en la introducción que la distribución geográfica en Conchucos para la tradición femenina del enrollado corresponde a la actual provincia de Huari. En Conchucos central, en la cuenca sur del río Yanamayo la actividad alfarera es masculina con el uso del paleteado complementando el enrollado. Esta última tradición se encuentra en las actuales provincias de Asunción, Fermín Fitzcarrald y Antonio Raimondi (fig. 3). Lo mismo se observa más al norte con el paleteado como técnica secundaria, en la región de Pomabamba (en Conopá y Shiullá), pero existen variaciones en cuanto a las denominaciones de las materias primas y herramientas utilizadas, lo que denota un sustrato lingüístico $-\mathrm{y}$ étnico- distinto 
(Druc, trabajo de campo en Pomabamba 2007, ms.). En los últimos años, Gabriel Ramón también ha trabajado en esta zona y seguramente que sus datos llevarán más informaciones al respecto.

Así, los tres marcadores étnicos previamente expuestos (tecnología, género del oficio, y distribución espacial de la tradición alfarera) sugieren la existencia de dos grupos socioculturales distintos en esta parte de Conchucos. Lo que nos lleva de nuevo a la cuestión de identificación del, o de los grupos en relación con estas tradiciones.

Inicialmente, había puesto en paralelo cada tradición con los grupos que supuestamente habitaban las regiones donde parecían vigentes, es decir, la tradición huarina con el grupo huari, y la tradición de San Luis-Chacas con el grupo Piscobambas que ocupaba las actuales provincias de Pomabamba y Mariscal Luzuriaga (fig. 3) (Druc, 2005; León Gómez, 2003). La frontera entre los dos grupos la constituye el río Yanamayo y su afluente occidental el río Yurma, en un eje este-oeste. Sin embargo, un examen detallado de la distribución de estas tradiciones muestra que no corresponde con este esquema, siendo el límite entre las dos tradiciones cerámicas más al sur del Yanamayo.

Además, hemos visto que estas tradiciones tienen una antigüedad que antecede la conquista inca de la zona. Entonces, ¿con qué grupo se identifica la tradición de la cuenca sur del Yanamayo?

\section{REALIDAD GEOGRÁFICA DE ICHOC HUARI Y ALLAUCA HUARI}

El término de parcialidad tiene una connotación geográfica. Las parcialidades del grupo Huari, Allauca Huari e Ichoc Huari, sugieren una parte derecha y una parte izquierda, respectivamente, en relación con algún elemento geográfico del territorio Huari.

Si nos fijamos en el estudio de León Gomez (2003) y en la lista de Roberto Levillier (De la Cruz Reyes et al., 1991: 30-32), donde anotaron cuál pueblo pertenecía a cuál parcialidad, podemos edificar un mapa de la distribución de las dos tradiciones alfareras en cuestión en el siglo XVI. En el mapa de la figura 4 vemos que el grupo huari abarcaba un territorio extenso, delimitado al norte por el río Yanamayo y al sur por el río Puchka, y coincide con las actuales provincias de Asunción, Fermín Fitzcarrald, Antonio Raymondi y parte de la provincia de Huari (León Gomez, 2003: 459). Mirando a un mapa más detallado se ve que las dos parcialidades de Huari son separadas por un ramo de cordillera atravesando el territorio Huari siguiendo un eje noreste al suroeste (fig. 4). Del lado oeste (o sea izquierdo en relación con este elemento topográfico) se encuentra Ichoc Huari. Del lado este, derecho, se extiende Allauca Huari. De allí hay la justificación de ichoc y allauca.

Así, la división del grupo Huari responde, por lo menos en parte, a una partición natural geográfica, un ramo de la Cordillera Blanca, que separa la cuenca sur del Yanamayo del valle del Huaritambo y de la región de Huari.

El territorio de cada parcialidad presenta «una unidad geográfica con áreas cultivables continuas», como lo nota Alexander Herrera (2003: 224) para la cuenca sur del Yanamayo. Esta unidad geográfica podría justificar la presencia de ayllus o grupos distintos en ambos lados de este ramo de cordillera, y el carácter diferente de las dos tradiciones observadas. El ramo de cordillera que separa los dos territorios (la cuenca del Yanamayo y la región de Huari) no es infranqueable, pero el cruce a pie, de Huari a Chacas toma unas 9 horas (29 kilómetros), pasando cerca del nevado Altar Mayor a 5586 metros de altura.

En conclusión, argumento que, en base a las diferencias en las tradiciones alfareras observadas y puesto que estas diferencias tienen raíces socioculturales, las parcialidades del grupo Huari están compuestas de llactas o ayllus diferentes y que el grupo Huari se formó a 


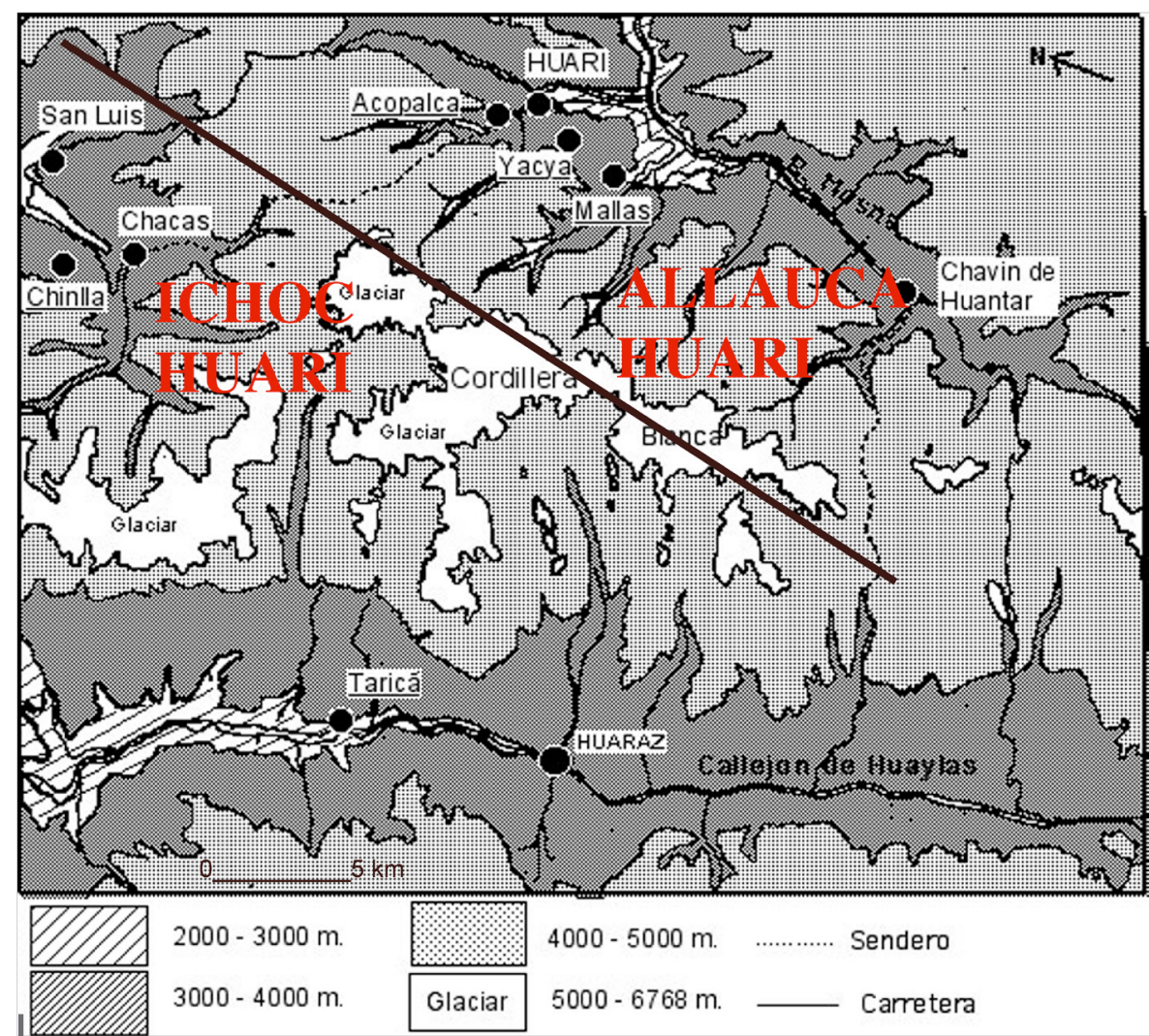

Figura 4 - Ubicación geográfica de las parcialidades de Huari.

Modificado a partir del mapa en Druc 2005, figura 2

base de dos grupos distintos. El género de un oficio y la tecnología de manufactura cerámica son elementos que se aprenden y se transmiten dentro de un grupo humano con mismos valores socioculturales. Además, la unidad geoespacial donde vive cada grupo refuerza la evolución autónoma de cada tradición, en este caso, de la producción cerámica.

En algún momento durante el Intermedio Tardío, estos grupos decidieron unirse. Esta nueva organización sociopolítica no significó el abandono de las respectivas tradiciones cerámicas. Con los cambios estructurales llevados a cabo por los españoles, el grupo Huari se desmanteló. Rostworowski (1990) nota que en general, las estructuras de base sufrieron menos reorganización y se observa que, para el caso de Conchucos central, hemos vuelto a una distribución geopolítica algo similar a la situación pre inca. Cabe notar que hace 30 años, los distritos de Chacas y San Luis (en la cuenca del Yanamayo) formaban parte de la provincia de Huari. Sin embargo, la dificultad de comunicaciones entre las dos regiones y la lejanía entre los distritos y la capital provincial (la ciudad de Huari), incitó una reorganización geopolítica, con la separación de estos distritos (Bebel Ibarra, comunicación personal, 2007), que ahora forman partes de nuevas provincias. Chacas se convirtió en la capital provincial de Asunción, San Luis en la capital de Fermín Fitzcarrald. 


\section{PARA UNA CORROBORACIÓN DE LA HIPÓTESIS DE TRABAJO}

La hipótesis de la existencia de dos grupos con tendencias socioculturales diferentes suele verificarse al nivel arqueológico en aspectos de la cultura material, en el conjunto de herramientas alfareras, en un análisis comparativo de los estilos decorativos. A esto se puede agregar el análisis de los conjuntos de vasijas (estilo y tecnología) en contextos residenciales como lo propone Janusek (2005) para identificar identidades sociales y el análisis de la organización del espacio doméstico y funcional como marcador de identidad etnica según Stanish (2005). Para el Intermedio Tardío, el conjunto de herramientas debería incluir el plato alfarero, paleta y broquel de arcilla cocida para Conchucos central (cuenca del Yanamayo), y plato solo para el sur de Conchucos (zona de Huari) además de las otras herramientas comunes (coronta de maíz, trapo, cuchillo, fragmento de mate). Pero esto supone que se encuentre todas las herramientas. El plato alfarero existe en los dos contextos (central y sur). Difiere un poco en diámetro, y a veces presenta una base anular en el sur, pero no siempre. Para poder ver estas diferencias a un nivel interpretativo regional, suelen encontrarse varios conjuntos. Un solo conjunto no es suficiente para sacar conclusiones. En cuanto a diferencias de género como marcador de identidad, funciona en contexto etnográfico, pero no se puede corroborar en contexto arqueológico.

Al nivel lingüístico, un examen de la toponimia local debería revelar nombres de apus, huacas y otros elementos referentes a ancestros míticos y origenes de las comunidades locales. Cabe notar que los vocablos utilizados para identificar a las materias primas en distintas partes de Conchucos y Callejón de Huaylas difieren. La arcilla o tierra arcillosa utilizada en las regiones de Huari y San Luis se llama «raaku» y la piedra que se muele y añade a la arcilla es el shashal. La mezcla resultante y lista para formar vasijas se llama «mito» en la zona de San Luis-Chacas. En la zona de Pomabamba, al contrario, el vocable mito refiere únicamente a la arcilla (y no a la mezcla) y la piedra negra que se muele y agrega se llama shilla. En el Callejón de Huaylas, se le denomina shashal a la piedra que se muele y agrega a la tierra arcillosa. Este «shashal no es una pizarra carbonífera negra como en Conchucos, sino un tipo de tierra compactada, gris y dura (los alfareros la califican de piedra —Druc, trabajo de campo, ms.-). En Conopá, utilizan la piedra shashal (pizarra carbonífera) para hacer crisoles. Estas variantes y diferencias permiten ver la necesidad de especificar y posiblemente analizar de que materiales se habla.

Al nivel etnográfico, valdría investigar cómo los habitantes se identifican en relación al «otro» (entre las comunidades de la región de Huari y las de la zona de Chacas y San Luis) y si se puede identificar el linaje de los alfareros. Cabe notar que los residentes de la zona de Huari ven diferencias en los vestidos, en los bailes, la comida y el quechua entre su zona y la de San Luis-Chacas. Tampoco parecen haber evidencias de casamientos entre personas de estas regiones en los registros de la parroquia de Huari (Julio Amaru, comunicación personal, 2007). Las reglas de endogamia de una llacta (al igual que para el ayllu) ${ }^{5}$ contribuyen a la conservación de las tradiciones dentro del grupo. Una llacta se distingue de otras llactas según niveles o grados de parentesco. El punto de partida lo define el umbral donde se permiten lazos de exogamia, o sea matrimonios afuera de la llacta (Frank Salomon, comunicación personal, 2007). Este punto o nudo relacional tiene una ubicación temporal en la historia de un grupo humano. Desde luego, Salomon sugiere investigar a qué nivel se sitúa el nudo relacional entre las dos parcialidades de Huari (Frank Salomon, comunicación personal, 2007).

Para reglas de parentesco relativas al ayllu, vease Zuidema (1977; 1989). 


\section{EVOLUCIÓN DE LAS TRADICIONES CERÁMICAS EN CONCHUCOS SUR Y CENTRAL}

Falta un aspecto en esta discusión, y es la dimensión histórica a la cual alude Wetherell (1982). Presenté en otro trabajo una discusión acerca de la antigüedad de las tradiciones cerámicas en Conchucos sur y central (Druc, 2005)6. Vale aquí ampliar la reflexión enfocándola hacia la evolución distinta de cada tradición anterior a la presencia inca. Veamos la situación para cada zona individualmente.

\section{1. Zona Huari}

Para la zona de Huari, de las investigaciones arqueológicas hechas por Bebel Ibarra (2003) se deduce que no hubo cambios mayores en los tipos de vasijas domésticas producidas desde el Intermedio Tardío. En el Intermedio Tardío, la ejecución de las vasijas es simple, sin mucho acabado y el repertorio de decoraciones es menor que en épocas anteriores. Más bien parece similar a lo que se practica todavía hoy. Sin embargo, se carece de estudios estilísticos de la cerámica colonial e histórica en esta zona, y es difícil por ahora pronunciarse sobre la cuestión de variaciones decorativas en las vasijas.

La tecnología del enrollado de la zona de Huari no parece haber sido alterada por influencias exteriores, durante o después del Intermedio Tardío. Un análisis reciente de fragmentos del Intermedio Tardío del sitio arqueológico de Marcajirca a ocho kilómetros de Acopalca y excavado por Bebel Ibarra muestra que el shashal era un componente mayor en la producción, al igual que la producción actual en la zona (Druc \& Ibarra, ms.). Desde luego, se puede imaginar que la tradición cerámica huarina podría remontar también al Horizonte Medio o ser oriunda de la región. Sin embargo, se necesita análisis de pasta de esta época para demostrarlo. Investigaciones de centros de producción, como Yacya, por ejemplo, ayudaría a entender mejor la profundidad histórica de esta tradición.

Una advertencia relativa a la implicación de las mujeres en el oficio, es la cuestión de la inmigración masculina durante la Colonia, y posiblemente anteriormente. La falta de hombres para varias tareas productoras y agrícolas muchas veces obliga a las mujeres a tomar las posiciones vacantes y hacer el trabajo que los hombres normalmente harían. Es un hecho que se observa en muchas partes del mundo. ¿Sería el caso para la zona de Huari? Tampoco se puede apartar el hecho de que la producción cerámica en esta región es un trabajo familiar, donde las mujeres manufacturan, pero los hombres se encargan de las otras tareas de producción. Una excepción se encuentra en Mallas, en la familia de Mercedes Varga. Mercedes cuenta que su padre, abuelo y tío hicieron cerámica. Pero aparece que aprendieron con su esposa, o en contexto de producción femenino, y no se pudo verificar hasta cuanto remontaba esta tradición familial.

Actualmente, la falta de hombres es un factor notable en la disminución o en el abandono de la producción. Influye sobre la cantidad e intensidad de producción. Es probable que en tiempos antiguos, la intensidad de producción y la participación masculina al oficio fueran superiores, inclusive con hombres produciendo también. Asimismo, otros pueblos en la zona hubieran podido ser centros de producción en el Intermedio Tardío y antes.

6 La versión inglesa (Druc, 2007) del libro sobre los alfareros de Conchucos ha puesto al día esta discusión. Para más detalles los lectores pueden referirse a esta nueva versión, digital, que incluye también un documental de 14 minutos. 


\section{2. Zona de la cuenca sur del Yanamayo, región San Luis-Chacas}

El paleteado es una tradición conocida como norteña probablemente desde el Horizonte Medio, que se extiende desde Piura al Jequetepeque, y más al sur (Bankes,1985; Camino, 1983; Cleland \& Shimada, 1994; Kroeber \& Muelle, 1942; Ravines, 1989). Gabriel Ramón (2008) examina esa tradición y su distribución geográfica con los conceptos de estilo técnico y diacronía. Observa variaciones en la distribución de los estilos técnicos y de las herramientas y propone una reinterpretación del material arqueológico, con posibilidad de desplazamiento de herramientas o de artesanos para explicar anomalías (e.g.la presencia de platos de alfareros típicos del sur en una zona norteña particular). Sin embargo, como lo nota Ramón (2005), el paleteado en Conchucos tiene diferencias con la técnica del norte del Perú. Las diferencias son tanto formales (con paletas más gruesas y contrapeso de piedra en el norte) como funcionales (uso estructural y decorativo en el norte, uso de construcción secundario para afirmar las paredes en Conchucos central).

A pesar de estas diferencias, existe la posibilidad de una influencia o de una transmisión de tecnología del norte del Perú a los Andes norcentrales, Ilegando a Ancash y al Callejón de Huaylas, donde esta técnica es también utilizada, en los valles costeños (e.g. el Santa) o en la sierra del departamento de la Libertad que limita con Conchucos. Si la hipótesis de una influencia del norte es correcta, significaría que aunque todos los elementos del paleteado norteño (formales como funcionales) no fueron adoptados, la noción del paleteado lo fue. Esta noción fue adaptada o superpuesta a la tradición existente en el norte y parte central de Ancash (Callejón de Huaylas, Conchucos central). Para invalidar esta hipótesis se necesitaría encontrar mediante excavaciones, talleres de cerámica o herramientas para la producción, como paletas y contrapesos (broqueles), que deberían fechar antes de su presencia en el norte.

Cabe notar que en el norte del Perú, como en el Callejón de Huaylas, en los valles costeños de Ancash y en Conchucos central, existe el paleteado como técnica primaria o secundaria de elaboración de las vasijas según las regiones. Los hombres son los productores dentro de un sistema de producción familial con participación de las mujeres para las tareas menores (Druc, 1996, 2005; Lavallée, 1967; Ravines, 1989)7.

Una cuestión queda relativa a la influencia posible sobre la producción cerámica en el Yanamayo de colones mitmas cañares o de Quito, trasladados a la región central de Conchucos por los incas (Herrera, 2003; 2006). Herrera sugiere que estos fueron traídos a Conchucos para trabajar en la edificación y mantenimiento del Capac Ñan y de sus tambos, en los campos y, a menudo, en las minas.

No se dice si habían alfareros entre estos colones. En Ecuador, en zona Cañar, la tradición cerámica es la del golpeado y en la región de Quito en la sierra norte, predominan las técnicas del molde y del molde invertido y acordelado (Sjöman, 1991; 1992). La técnica del golpeado es una técnica secundaria de elaboración de las vasijas, utilizada para sacar el «pecho» una vez formada la vasija, jalando el barro (Sjöman, 1991: 67-68). Se usan dos golpeadores o huactanas hechas de arcilla quemada en forma de platillo con base, una convexa para empujar o golpear las paredes desde el interior, la otra cóncava o plana para los contragolpes por el exterior (Sjöman, 1991: 67-68). Son las mujeres que se encargan

7 Véase Druc, 2007 para una excepción a la regla, donde una mujer, contratada por un alfarero de Taricá, en el Callejón de Huaylas, ayudó éste a producir cuando estuvo enfermo. Sin embargo, se considera que es un trabajo de hombre y que las mujeres se pueden enfermar a trabajar el barro (Sra. Olimpia Norabueno León, esposa del alfarero de Taricá, Nicanor Saavedra, comunicación personal, 2007). 
de todo el proceso de producción, incluida la quema. Se elabora la vasija sobre otra vasija invertida. Esta técnica sería anterior a la llegada de los incas en la región (Sjöman, 1991: 67-68).

De lo expuesto arriba, deducimos que las técnicas prevalentes en Ecuador no fueron implementadas en Conchucos y que no influenciaron las tradiciones locales.

Terminaré esta discusión sobre el origen y la evolución posible de la tradición cerámica en Conchucos central con una doble propuesta de pista de investigación: a) la tradición cerámica en la zona de San Luis-Chacas en algún momento de su historia fue influenciada por elementos foráneos a la zona (el paleteado norteño) siguiendo contactos con el norte; o b) un grupo emigrante de individuos o de alfareros se estableció en la zona, trayendo consigo la tradición del paleteado y el carácter masculino del oficio.

El esquema de la figura 5 resume esta discusión, presentando una perspectiva diacrónica de las tradiciones cerámicas en relación con la organización sociopolítica de los grupos de la zona de estudio (fig. 5).

\section{CONCLUSIÓN}

Este estudio presentó los argumentos que permitieron proponer la existencia de dos grupos distintos en el sur y en el centro de Conchucos. Las tradiciones cerámicas en cada zona (región de Huari y cuenca sur del Yanamayo) muestran diferencias importantes en su tecnología y manera de producción, sugiriendo una evolución cerámica distinta dentro de comunidades diferentes.
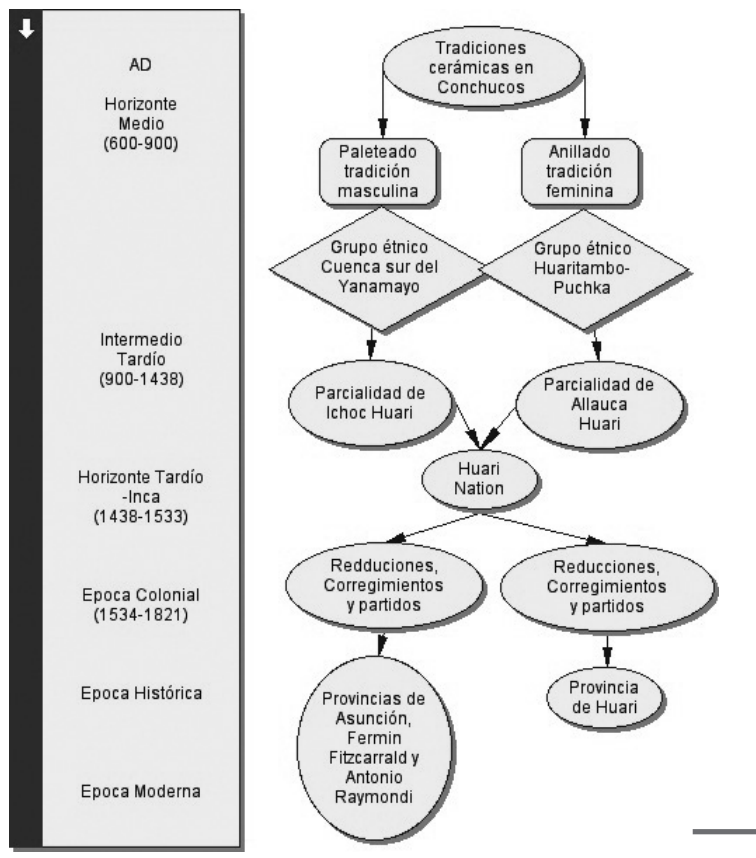

Figura 5 - Perspectiva diacrónica de la situación en Conchucos sur y central en relación con las tradiciones cerámicas.

Las fechas cronológicas toman en consideración las secuencias vigentes para Conchucos (Márquez Zorrilla, 1998; Matos Colchado, 2000; Ibarra, 2003; Lau, 2003). El año 1821 marca la independencia del Perú, pero también la formación inicial del departamento de Ancash por San Martin (bajo el nombre de Huaylas, que fue cambiado a Ancash en 1837) 
En documentos del siglo XVII los pueblos en donde se produce ahora la cerámica, figuran como parte de una u otra mitad del grupo Huari. El presente análisis relacionando tradición cerámica y grupos humanos muestra que la ubicación geográfica de las parcialidades de Ichoc Huari y Allauca Huari sigue una partición oeste-este (izquierda-derecha) y corresponde a la presencia de dos grupos distintos, moradores de las respectivas regiones del valle de Huari y de la cuenca sur del Yanamayo. No se excluye, a un nivel superior, una base de creencias comunes, con una base lingüística y socio-cultural distinta, y diferencias en la organización profesional y en los estilos técnicos alfareros.

La discusión sobre el concepto de etnia para esta zona concluye en una organización pluriétnica - o pluri-ayllus - del grupo Huari y rechaza la existencia de una macroetnia. Sin embargo, el vocablo de etnia o grupo étnico no logra rendir bien la realidad andina.

Los datos etnográficos y de cerámica sugieren la existencia de grupos distintos anteriormente a la formación de la nación Huari, con tradiciones cerámicas diferentes, posiblemente remontándose al Horizonte Medio.

Por último, este artículo ofrece orientaciones de investigación para corroborar la hipótesis presentada y evaluar la profundidad y la variabilidad de las tradiciones cerámicas en Conchucos, de modo diacrónico y sincrónico. Cabe notar que hay poca información sobre la producción cerámica para la zona norte de Conchucos. Rogger Ravines cita unos comentarios relacionados en su recopilación de 1989, sin más corroboración. El patrón de distribución de las tradiciones cerámicas en Conchucos y el Callejón de Huaylas que se desprende del presente estudio sugiere, sin embargo, que la producción en el norte consta de una tradición similar a lo encontrado en la región del Yanamayo. Trabajos personales de campo en 2007 sustentan esta suposición, aunque haya variaciones importantes en la denominación de las materias primas como se ha discutido más arriba.

En el aspecto teórico de la discusión sobre grupos étnicos en el contexto de Conchucos aún falta mucho trabajo por realizar. Espero que esta carencia impulsará más estudios. En particular, sería importante revisar los datos etnográficos acerca de la presencia de tecnología cerámica distinta en la misma región y si esta diferencia está atribuida a la presencia de grupos étnicos distintos, a la presencia de alfareros inmigrantes o a una complementariedad económica. Un buen ejemplo de diversidad étnica en la misma región, revelada por el estilo técnico de cada tradición cerámica, aunque fuera de la esfera andina, se encuentra en Mali (Gallay et al., 1996) y Camerún (Gosselain, 2002).

En Mali, el estudio etnoarqueológico hecho por Alain Gallay et al. (1996) tiene un uso directo en la identificación de las varias etnias presentes en los sitios arqueológicos.

Las investigaciones arqueológicas que se llevaron a cabo en los últimos diez años en Conchucos por Orsini (2003; 2006), Herrera (2003; 2006), Alexis Mantha (2006) e Ibarra (2003; 2006), entre otros, generaron una fuente de datos y de material cerámico importante. Hace falta ahora relacionar y sintetizar los estudios individuales con fines de brindar una interpretación más global de la producción cerámica en esta parte de la sierra del Perú.

\section{Agradecimientos}

Agradezco a varios colegas por haberme alentado a ir más allá de los conceptos expresados en este artículo. En particular quiero agradecer a George Lau para sus comentarios y la revisión detallada del texto inicial y a Frank Salomon, Tom Zuidema y Bebel Ibarra que me obligaron a reexaminar los conceptos de identidad social y a clarificar mi texto. Cualquier error de interpretación es mío. Agradezco también a Beatriz De Las Casas para la lectura del manuscrito. 


\section{Referencias citadas}

ABERCROMBIE, T., 1991 - Articulación doble y etnogénesis. In: Reproducción y transformación de las sociedades andinas siglos XVI-XX (S. Moreno Y. \& F. Salomon, eds.): 197-212; Quito: Ediciones Abya-Yala: Movimiento Laico para América Latina.

ACEVEDO, S., 2004 - La loza de la tierra: cerámica vidriada en el Perú. In: La loza de la tierra (S. Acevedo, ed.): 16-39; Lima: Instituto Cultural Peruano Norteamericano (ICPNA), Universidad Ricardo Palma (URP).

BANKES, G., 1985 - The Manufacture and Circulation of Paddle and Anvil Pottery on the North Coast of Peru. World Archaeology, 17: 270-277.

CAMINO, L., 1983 - Los últimos olleros de Sinsicap. Boletín de Lima, 29: 31-36.

CLELAND, K. M. \& SHIMADA, I., 1994 - Ceramios paleteados: Tecnología, esfera de producción y sub-cultura en el Perú antiguo. In: Tecnología y organización de la producción de cerámica prehispánica en los Andes (I. Shimada, ed.): 321-348; Lima: Fondo Editorial de la Pontificia Universidad Católica del Perú.

COHEN, R., 1978 - Ethnicity: «Problem and Focus in Anthropology». Annual Reviews in Anthropology, 7: 379-403.

D'ALTROY, T. N., LORANDI, A. M. \& WILLIAMS, V., 1994 - Producción y uso de cerámica en la economía política inca. In: Tecnología y organización de la producción de cerámica prehispánica en los Andes (I. Shimada, ed.): 395-441; Lima: Fondo Editorial de la Pontificia Universidad Católica del Perú.

DE LA CRUZ REYES, H., SAMANIEGO ROMÁN, L., REYES BLACIDO, P., DE LA CRUZ REYES, R. N. \& DE LA CRUZ REYES, F. E., 1991 - Olleros en la Historia regional de Ancash, 103 pp.; Lima: Centro de Elaboración de Material Educativo de la Universidad Nacional de Educación (CEMED-UNE)

DRUC, I. C., 1996 - De la etnografía hacia la arqueología: aportes de entrevistas con ceramistas de Ancash (Perú) para la caracterizazión de la cerámica prehispánica. Bulletin de l'Institut Français d'Études Andines, 25 (1): 17-41.

DRUC, I. C., 2000 - Shashal o no shashal, esa es la cuestión. Etnoarqueología cerámica en la zona de Huari, Ancash. Bulletin de I'Institut Français d'Études Andines, 30 (1): 157-173.

DRUC, I. C., 2001 - Soil Sources for Ceramic Production in the Andes. In: Archaeology and Clays (I. Druc, ed.): 95-105; Oxford: British Archaeological Reports S942.

DRUC, I. C., 2005 — Producción alfarera y etnoarqueología en Conchucos, Ancash, Perú, 110 pp.; Lima: Instituto Cultural Runa.

DRUC, I. C., 2007 - Encounters with Andean Potters; 109 pp.; El Cajón (California): National Social Sciences Press (NSSP).

DRUC, I. C. \& IBARRA ASENCIOS, B., ms. - Résultats préliminaires des analyses céramiques du site de Marcajirca, Ancash, Pérou.

DUVIOLS, P., 1973 - Huari y Llacuaz, agricultores y pastores: dualismo prehispánico de oposición y complementariedad. Revista del Museo Nacional, 39: 153-191.

DUVIOLS, P., 1986 - Cultura andina y represión: procesos y visitas de idolatrías y hechicerías, Cajatambo, siglo XVI, 570 pp.; Lima: Centro de Estudios Rurales Andinos «Bartolomé de las Casas». 
ESPINOZA SORIANO, W., 1978 - Huaraz: poder, sociedad y economía en los siglos XV y XVI. Reflexiones en torno a las visitas de 1558 y 1712, 165 pp.; Lima: Universidad Nacional Mayor de San Marcos, Seminario de Historia Rural Andina.

GALLAY, A., HUISECOM, E., MAYOR, A. \& DE CEUNINCK, G., 1996 - Hier et aujourd'hui: Des poteries et des femmes. Céramiques traditionnelles du Mali, 142 pp.; Genève: Département d'anthropologie et d'écologie, Université de Genève.

GOSSELAIN, O. P., 2002 - Poteries au Cameroun méridional. Styles techniques et rapports à l'identité, 254 pp.; París: CNRS Éditions.

HAGSTRUM, M., 1987 - Supply and Demand of Ceramic Products: An Ethnoarchaeological Study of Community Specialization in the Central Andes, Peru. Presentación de $52^{\text {nd }}$ Meeting of the Society of American Archaeology, Toronto, Canada.

HAYASHIDA, F., 1998 - New Insights into Inka Pottery Production. In: Andean Ceramics: Technology, Organization, and Approaches (I. Shimada, ed.): 313-335; Philadelphia: University of Pennsylvania. MASCA Research Papers in Science and Archaeology Vol. 15 Supplement.

HERNÁNDEZ PRÍNCIPE, R., 1923 - Mitologia andina. Revista Inca, 1(1): 25-78.

HERRERA WASSILOWSKY, A., 2003 - Patrones de asentamientos y cambios en las estrategias de ocupación en la cuenca sur del río Yanamayo, Callejón de Conchucos. In: Arqueología de la sierra de Ancash (B. Ibarra Asencios, ed.): 220-249; Lima: Instituto Cultural Runa.

HERRERA WASSILOWSKY, A., 2006 - Territorio e identidad: apuntes para un modelo de la complejidad social andina. In: La complejidad social en la Sierra de Ancash: ensayos sobre paisaje, economía y continuidades culturales (A. Herrera Wassilowsky, C. Orsini \& K. Lane, eds.): 3-18; Milano: Civiche Raccolte d'Arte Applicata del Castello Sforzesco.

IBARRA ASENCIOS, B., 2003 - Arqueología del valle del Puchca. In: Arqueología de la Sierra de Ancash (B. Ibarra Asencios, ed.): 252-330; Lima: Instituto Cultural Runa.

IBARRA ASENCIOS, B., 2006 - Ancestros y muerte durante la época prehispánica en la sierra de Ancash: buscando nuestros antepasados. In: La complejidad social en la sierra de Ancash: ensayos sobre paisaje, economía y continuidades culturales (A. Herrera Wassilowsky, C. Orsini \& K. Lane, eds.): 85-100; Milano: Civiche Raccolte d'Arte Applicata del Castello Sforzesco.

JANUSEK, J. W. 2005. - Of Pots and People: Ceramic Style and Social Identity in the Tiwanaku State. In: Us and Them: Archaeology and Ethnicity in the Andes (R. Martin Reycraft, ed.): 34-53; Los Angeles: The Cotsen Institute of Archaeology, University or California, Monograph 53.

KROEBER, A. L. \& MUELLE, J. C., 1942 - La cerámica paleteada de Lambayeque. Revista del Museo Nacional, XI (1): 1-24; Lima.

LAU, G. F., 2003 - Evidencias radiocarbónicas para el estudio de las transformaciones culturales en Recuay. In: Arqueología de la sierra de Ancash (B. Ibarra Asencios, ed.): 135-159; Lima: Instituto Cultural Runa.

LAVALLÉE, D., 1967 - La poterie d'Aco (Andes centrales du Pérou). Objets et Mondes, VII (2): 103-120.

LECHTMAN, H., 1977 - Style in Technology. Some Early Thoughts. In: Material Culture: Styles, Organization, and Dynamics of Technology $(\mathrm{H}$. Lechtman \& R. S. Merrill, eds.): 3-20; St. Paul: Proceedings of the American Ethnological Society.

LEÓN GÓMEZ, M., 2003 - Espacio geográfico y organización social de los grupos étnicos del Callejón de Conchucos durante los siglos XVI y XVII. In: Arqueología de la sierra de Ancash (B. Ibarra Asencios, ed.): 457-466; Lima: Instituto Cultural Runa. 
Tradición alfarera, identidad social y el concepto de etnias tardías en Conchucos, Ancash, Perú

LEROI-GOURHAN, A., 1988 - Dictionnaire de la préhistoire; París: Presses Universitaires de France.

MANTA, A. 2006 - Late Prehistoric Social Complexity in the Rapayán Valley, Upper Marañon Drainage, Central Andes of Peru. In: La complejidad social en la sierra de Ancash: ensayos sobre paisaje, economía y continuidades culturales (A. Herrera Wassilowsky, C. Orsini \& K. Lane, eds.): 35-61; Milano: Civiche Raccolte d'Arte Applicata del Castello Sforzesco.

MÁRQUEZ ZORRILLA, S., 1998 - Huari y Conchucos, 137 pp.; Lima: Ediciones Opulares. Tercera edición.

MATOS COLCHADO, S., 2000 - Huaylas y Conchucos en la historia regional, 318 pp.; Lima: Editorial San Marcos.

ORSINI, C., 2003 - Transformaciones culturales durante el intermedio temprano en el valle de Chacas. In: Arqueología de la sierra de Ancash (B. Ibarra Asencios, ed.): 162-173; Lima: Instituto Cultural Runa.

ORSINI, C., 2006 - ¿Metáforas de complejidad social? Huari, Llacuaz, organización del territorio y especialización económica en Chacas (valle del Chacapata Perú). In: La complejidad social en la sierra de Ancash: ensayos sobre paisaje, economía y continuidades culturales (A. Herrera Wassilowsky, C. Orsini \& K. Lane, eds.): 151-163; Milano: Civiche Raccolte d'Arte Applicata del Castello Sforzesco.

RAMÓN, G., 2005 - Reseñas. Bulletin de I'Institut Français d'Études Andines, 34 (3): 480-485

RAMÓN, G., 2008 - Producción alfarera en Piura (Perú): estilos técnicos y diacronía. Bulletin de I'Institut Français d'Études Andines, 37 (3): 477-509.

RAVINES, R., 1989 - Principales comunidades y centros alfareros del Perú. In: La cerámica tradicional del Perú (R. Ravines \& F. Villiger, eds.): 45-57; Lima: Los Pinos.

ROSTWOROWSKI DE DIEZ CANSECO, M., 1981 - La voz parcialidad en su contexto de los siglos XVI y XVII. Etnohistoria y Antropología Andina: 35-47; Lima: Segunda Jornada del Museo Nacional de Historia.

ROSTWOROWSKI DE DIEZ CANSECO, M., 1990 - Las macroetnias en el ámbito andino. Allpanchis, 35-36: 3-28.

ROSTWOROWSKI DE DIEZ CANSECO, M., 1996 - Estructuras andinas del poder, 202 pp.; Lima: Instituto de Estudios Peruanos. Cuarta edición.

SACKETT, J. R., 1990 - Style and Ethnicity in Archaeology: the Case for Isochrestism. In: The Uses of Style in Archaeology (M. Conkey \& C. Hastorf, eds.): 32-43; Cambridge: Cambridge University Press.

SAIGNES, T., 1991 - Lobos y ovejas. Formación y desarrollo de los pueblos y comunidades en el sur Andino (Siglos XVI-XX). In: Reproducción y transformación de las sociedades Andinas siglos XVI-XX (S. Moreno Y. \& F. Salomon, eds.): 91-135; Quito: Ediciones Abya-Yala: Movimiento Laicos para América Latina.

SALAZAR, J. M., 1998 - Social Identity and National Identity. In: Social Identity (S. Worchel, J. Francisco Morales, D. Páez \& J.-C. Deschamps, eds.): 114-123; London: Sage Publications.

SJÖMAN, L., 1991 - Cerámica popular: Azuay y Cañar, 121 pp.; Cuenca: Centro Interamericano de Artesanías y Artes Populares (CIDAP).

SJÖMAN, L., 1992 - Vasijas de barro. La cerámica popular en el Ecuador, 406 pp.; Cuenca: Centro Interamericano de Artesanías y Artes Populares (CIDAP). 
STANISH, C. 2005. - Migration, Colonies, and Ethnicity in the South-Central Andes. In: Us and Them: Archaeology and Ethnicity in the Andes (R. Martin Reycraft, ed.): 226-232; Los Angeles: The Cotsen Institute of Archaeology, University or California, Monograph 53.

TAYLOR, G., 1980 - Rites et traditions de Huarochirí, 243 pp.; París: L’Harmattan.

TSCHOPIK, H., 1950 - An Andean Ceramic Tradition in Historical Perspective. American Antiquity, 15: 196-218.

URTON, G., 1991 - Las unidades de análisis en el estudio de la reproducción y transformación de las sociedades andinas. In: Reproducción y transformación de las sociedades Andinas siglos XVI-XX (S. Moreno Y. \& F. Salomon, eds.): 29-46; Quito: Ediciones Abya-Yala: Movimiento Laicos para América Latina.

VELDE, B. \& DRUC, I., 1999 - Archaeological Ceramic Materials, 299 pp.; Berlin: Springer Verlag.

WETHERELL, N., 1982 - Cross Cultural Studies of Minimal Groups: Implications for the Social Identity Theory of Intergroup Relations. In: Social Identity and Intergroup Relations (H. Tajfel, ed.): 207-240; Cambridge: Cambridge University Press.

WIESSNER, P., 1990 - Is there a unity to style? In: The Uses of Style in Archaeology (Margaret Conkey \& Christine Hastorf, eds.): 105-112; Cambridge: Cambridge University Press.

ZUIDEMA, R. T., 1973 - La parenté et le culte des ancêtres dans trois communautés: un compte rendu de 1622 par Hernández Príncipe. Recherches Amérindiennes au Québec, 3 (1-2): 129-146.

ZUIDEMA, R. T., 1977 - The Inca kinship system: A New Theoretical View. In: Andean Kinship and Marriage (Ralph Bolton \& Enrique Mayer, eds.): 240-292; Washington, D.C.: American Anthropological Association.

ZUIDEMA, R. T., 1989 - Reyes y guerreros, 563 pp.; Lima: Fomciencias. 